\title{
Biomarker Identification from RNA-Seq Data using a Robust Statistical Approach
}

\author{
Zobaer Akond1, 2, 4, , Munirul Alam², Md. Nurul Haque Mollah ${ }^{3}$ \\ ${ }^{1}$ Agricultural Statistics and Information \& Communication Technology (ASICT) Division, Bangladesh Agricultural Research Institute \\ (BARI), Joydebpur, Gazipur-1701, Bangladesh; 2Institute of Environmental Science, University of Rajshahi-6205, Bangladesh; \\ ${ }^{3}$ Emerging Infections, Infectious Diseases Division, International Centre for Diarrheal Disease Research, Bangladesh (icddr,b); \\ ${ }^{4}$ Bioinformatics Lab, Department of Statistics, University of Rajshahi, Rajshahi-6205, Bangladesh; Zobaer Akond - E-mail: \\ akond25@yahoo.com; *Corresponding author
}

Received March 5, 2018; Revised April 2, 2018; Accepted April 5, 2018; Published April 30, 2018

doi:10.6026/97320630014153

\begin{abstract}
:
Biomarker identification by differentially expressed genes (DEGs) using RNA-sequencing technology is an important task to characterize the transcriptomics data. This is possible with the advancement of next-generation sequencing technology (NGS). There are a number of statistical techniques to identify DEGs from high-dimensional RNA-seq count data with different groups or conditions such as edgeR, SAMSeq, voom-limma, etc. However, these methods produce high false positives and low accuracy in presence of outliers. We describe a robust t-statistic method to overcome these drawbacks using both simulated and real RNA-seq datasets. The model performance with $61.2 \%, 35.2 \%, 21.6 \%, 6.9 \%, 74.5 \%, 78.4 \%, 93.1 \%, 35.2 \%$ sensitivity, specificity, MER, FDR, AUC, ACC, PPV, and NPV, respectively at $20 \%$ outliers is reported. We identified $409 \mathrm{DE}$ genes with $p$-values $<0.05$ using robust t-test in HIV viremic vs avirmeic state real dataset. There are 28 up-regulated genes and 381 down-regulated genes estimated by log2 fold change (FC) approach at threshold value 1.5. The up-regulated genes form three clusters and it is found that 11 genes are highly associated in HIV1/AIDS. Protein-protein interaction (PPI) of up-regulated genes using STRING database found 21 genes with strong association among themselves. Thus, the identification of potential biomarkers from RNA-seq dataset using a robust $t$-statistical model is demonstrated.
\end{abstract}

Keywords: RNA-seq data, differentially expressed genes, robust t-statistic, gene-disease network, protein-protein interaction.

\section{Background:}

Transcriptomics is an evolving and continually growing field of Bioinformatics for biomarker discovery [1]. RNA-sequencing (RNA-seq) technology is an important branch of transcriptomics for identification of novel genes. This technique helps to identify the differentially expressed genes or transcripts (DEGs) associated to trait of interest from the voluminous transcriptomic data. Previously microarray technology had been used by the biological and biomedical researchers for discovering the candidate genes and differentially expressed markers between two or more groups of interest. In recent years, huge amount of transcriptomic data can be generated using high-throughput next-generation sequencing (NGS) technology of cDNA (RNAseq), which ultimately yield RNA-seq count data for subsequent analysis [6]. Additionally, this approach includes the identification of disease biomarkers that may be important in the diagnosis of the different types and subtypes of diseases, with several implications in terms of prognosis and therapy [2]. This ISSN 0973-2063 (online) 0973-8894 (print) sequence-based technology has created significant scope of studying the transcriptome by enabling a wide range of novel applications, including detection of alternative splicing isoforms [3-4], detecting novel genes, gene promoters, isoforms, and allelespecific expression [5]. RNA-seq uses NGS technology to sequence cDNA that has been derived from an RNA sample, and hence generates millions of short reads [6]. These reads are then usually mapped to a reference genome and the number of reads mapping within a genomic feature of interest (such as a gene or an exon) is used as a measure of the abundance of the feature in the analyzed sample [6]. One important objective for RNA-seq is to identify DEGs under different conditions. Researchers typically target for differential expression analysis called "count matrix", where each row represents the gene (or exons or genomic loci), each column represents the sample, and each cell indicates the number of reads mapped to the gene in the sample [7]. A basic research problem in many RNA-seq analyses is the discovery of DEGs between different sample groups (e.g. healthy

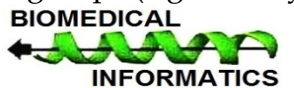


and disease). RNA-seq analysis has some benefits over microarrays for DE analysis including wide dynamic range and a lower background level, and the chance to detect and quantify the expression of previously unknown transcripts [8-10]. To deal with the increasing popularity of RNA-seq technology, several statistical tools have been developed so far by the researchers and these are being continuously updated for robust data analysis. Most of the computational methods such as edgeR [11] are based on negative binomial models. SAMseq [12] is a non-parametric method. Gene-level read counts transformation-based method is voom-limma [14]. Still it is important to keep in mind that even these methods are based on an assumption that most genes are equally expressed in the samples, and that the differentially expressed genes are divided more or less equally between upand down-regulation [2]. For detection of DE genes edgeR, SAMSeq and voom-limma are the popular methods. However these methods are sensitive to outliers. In this study we propose a new method robust $t$-statistic using minimum $\beta$-divergence method [15].

\section{Methodology:}

RNA-seq count data often produce noisy data called outliers during data generating steps. Presence of such outliers in the particular dataset will surely provide misleading results in the downstream analysis. There is however a number of statistical tools for identification of over-expressed and under-expressed genes, but they are sensitive to outliers in some cases. In this investigation we considered edgeR, SAMSeq and voom-limma the proposed procedures for performance analysis with the help of synthetic and real dataset (Figure 8).

\section{Robust t-statistic for Biomarker Detection (Proposed):}

The two sample-sample $t$-test statistic depends on the mean $(\mu)$ and variance $\left(\sigma^{2}\right)$ of the samples. The classical mean and variance are very much sensitive to outliers may mislead detection of DEGs. So, we used robust mean and variance with the minimum $\beta$-divergence method [15] instead of classical estimators. For robust $t$-statistic the classical estimators are replaced by the $\beta$ estimators. The proposed method tolerate outliers using the $\beta$ weight function, it is also used as a weight for mean and variance estimators [15]. It is an iterative method for estimating mean and variance estimators for calculating $t$-statistic values. The performance of the proposed method depends on the tuning parameter $\beta$, the optimum value for $\beta=0.2$ was selected using cross validation method [15].

\section{Data Transformation:}

To transform the RNA-Seq expression data we used the z- score transformation for making the data belong normalized data family. In this study $\mathrm{Z}$ normalized score values were used for the normalization with the mean $(g)$ and the standard deviation $S D$ $\left(g_{i}\right)$ of the $i^{\text {th }}$ genes. The normalized data is used to detect the DE genes using robust $t$-statistic. The fold change (FC) $\log 2$ approach used for selection of up-regulated and down-regulated genes with 1.5 threshold value [16]. The steps for identifying up and down regulated genes from gene expression data (GED) are shown

(Figure

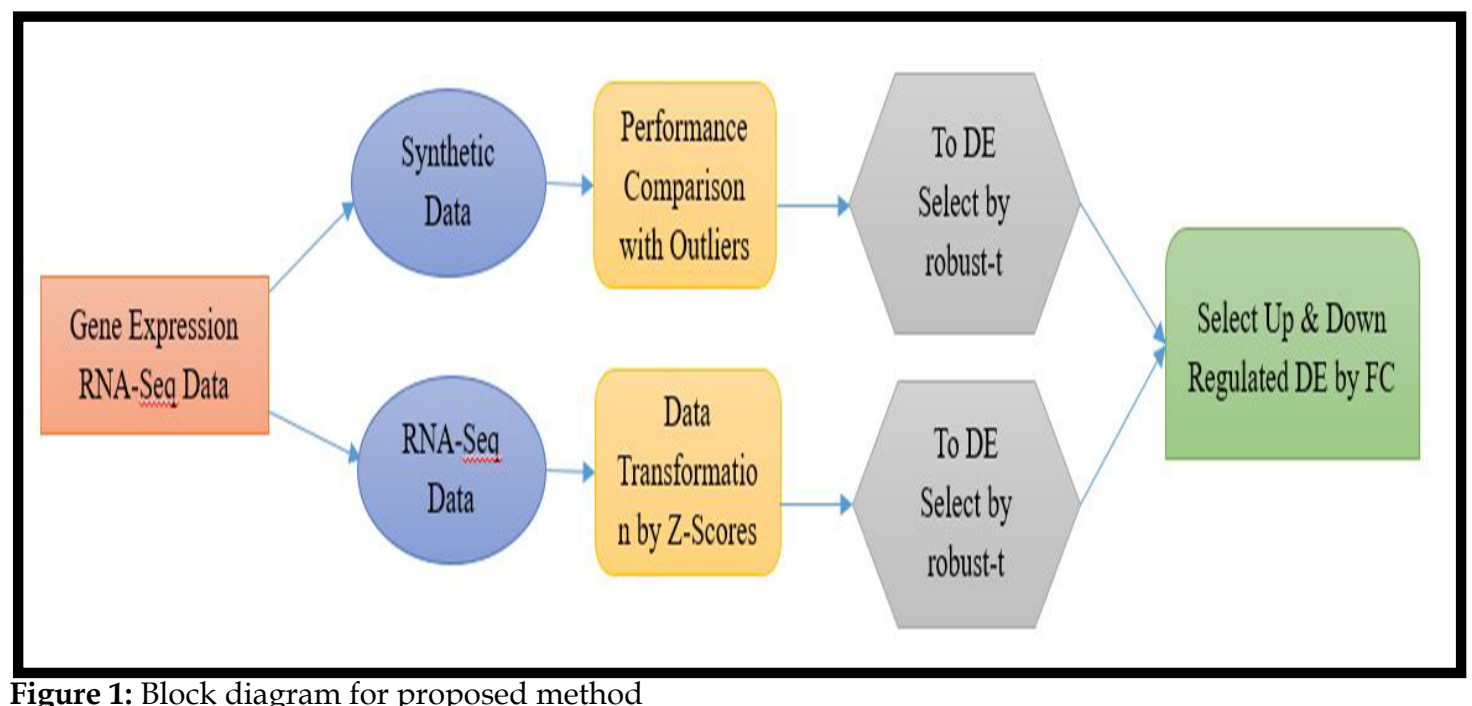

Figure 1: Block diagram for proposed method

\section{Performance Evaluation:}

We need statistical indices/measures to estimate the performance of different DE gene identification methods for binary classification tests such as healthy and disease. Outcomes are always divided into four categories for binary classification such as (i) healthy samples are correctly predicted as healthy termed as true positive (TP), (ii) healthy sample are incorrectly predicted as disease termed as false negative (FN), (iii) disease samples are ISSN 0973-2063 (online) 0973-8894 (print) correctly predicted as disease termed as true negative (TN) and (iv) disease samples are incorrectly predicted as healthy which is called false positive (FP) in Table 1 . We then calculate the following performance indices/measures using the following confusion matrix as follows: True Positive Rate (TPR) or Sensitivity $=n T P /(n T P+n F N)$, True Negative Rate $(T N R)$ or Specificity $=n T N /(n T N+n F P)$, False Positive Rate $(F P R)=$ 


\section{Open access}

$\mathrm{nFP} /(\mathrm{nFP}+\mathrm{nTN})$, False Negative Rate $(\mathrm{FNR})=\mathrm{nFN} /(\mathrm{nFN}+\mathrm{nTP})$ and False Discovery Rate $(\mathrm{FDR})=\mathrm{nFP} /(\mathrm{nTP}+\mathrm{nFP})$.

\section{Results and Discussions:}

All methods were run on the same dataset and evaluated the statistical performance indices/measures such as ROC, AUC, pAUC, MER and FDR. The performance measure AUC was computed for each of the statistical methods using open source $\mathrm{R}$ package ROC. All $\mathrm{R}$ packages are freely available in the comprehensive $\mathrm{R}$ archive network (https://www.cran.rproject.org) and bioconductor (https://www.bioconductor.org).

\section{Synthetic Study:}

The synthetic counts data were generated for each gene from a Negative Binomial distribution. It controls the settings and the true differential expression status of each gene. We generated gene expression profiles of $\mathrm{G}=1000$ genes of $\mathrm{k}=2$ groups (healthy and disease) each with $\mathrm{n} 1=\mathrm{n} 2=3$ samples. Among the expressions of 1000 genes, we divided these expressions into two groups (expressions of important genes or DE genes, 100 and expressions of the unimportant genes or EE genes, 900). In order to determine the performance of the robust $t$-test in comparison of the three well-known DE genes identification methods (edgeR, SAMSeq, voom+limma) of RNA-Seq data, we investigated the performance of all fours methods in presence of outliers using the simulated dataset $(G=1000)$ where $10 \%$ of the genes are DE genes. A method is said to be comparatively good performer if it yields larger values of TPR, TNR, AUC and small value of FDR. To demonstrate the effect in presence of outliers for performance evaluation of these DE genes identification methods, we randomly introduce $5 \%, 10 \%, 15 \%$ and $20 \%$ genes are corrupted by outliers. (Figure 2a-2d) shows the boxplot of AUC values of the four methods estimated from the simulated data at different outlier levels. It showed that the AUC of our proposed method is high. It is observed from the Table 2 that the AUC values of proposed method are $0.75,0.71,0.74$ and 0.75 for $5 \%, 10 \%, 15 \%$ and $20 \%$ respectively. The proposed method provided the low FDR and high ROC with outliers (Figure 3 and 4). Identification of DEGs is vital issues for personalised medicine and drug discovery. From Table 2 the proposed method shows low MER (misclassification error rate) than the edgeR, SAMSeq and voomlimma methods.

\section{Real RNA-Seq Data Analysis:}

The RNA-Seq data GSE5220 was collected from the GEO database (https:// www.ncbi.nlm.nih.gov/geo/query/acc.cgi?acc=GSE522 0) [17]. It contains $16 \mathrm{HIV}$ infected patients (8 patients are pre and 8 are post HAART cessation) and 22283 genes. It is observed that the gene expression changes in monocytes as a result of rebound of HIV viral load. From the HIV viremic vs avirmeic state real dataset we identified 409 DE genes at $5 \%$ level of significance with $p$-values $<0.05$ using robust $\mathrm{t}$-test. There are 28 up-regulated genes and 381 down regulated genes by log2 fold change (FC) approach with threshold value 1.5 from the RNA-Seq count data [16]. The molecular networks of the selected up-regulated genes are visualized using open source bioinformatics software platform Cytoscape (version 3.6.0) [18], the gene-disease network shows the interaction among genes and HIV disease. Figure 5a showed that there are 11 up-regulated genes (MDFIC, CDK14, SRP72, HGF, CHRDL1, MLX, AKR7A3, THAP11, DUSP3, ELL2, and FGFR1), which are highly correlated that are more interaction is existed with the HIV than the other genes. Figure $5 \mathbf{b}$ showed the hierarchical clustering of 28 up-regulated genes, which formed 3 different gene major functional groups. The group-I (DUSP3, INSR, PRPF31, SKAP2, MDFIC, CCL2, SIOOA14, ZNF35, THAP11, CHRDL1) genes are significant for the biological process in the cell, group-II genes (GCM1, FGFR1, GLT8D2, CDK14, G PS1, MLX, ELL2, STAP2, RNF39, PGPEP1, HGF) are insulin receptor complex, COP9 signalosome and insulin receptor complex and group-III genes (AKR74A3, PEBPI, TPP1, SNRK, CAPN11, SRP72, C18orf25) are proteosome component (PCI) domain composition. From the Figure $5 c$ the 381 down-regulated genes are detected by the proposed method. There are 10 genes (IGK, PRKAB2, SIGLEC1, APBB2, PRKCA, SPTBN1, PART1, ZNF764, TBC1D5, and RNASEH2B), which are highly correlated with the HIV disease and possess strong interaction with HIV disease. The protein-protein interaction of up-regulated genes analysis using STRING (version 10.5) database [19] produced three functional clustering groups (Figure 6a-6b) using k-means clustering algorithm. There are 21 genes showed the strong association among them (Figure 6a) and 20 genes are structured proteins. The MDFIC gene is the unstructured protein and it helps to make strong bond among other genes. The PPI network among 381 down-regulated genes is strong which forms three functional groups using k-means clustering algorithm (Figure 6c-6d). There are 45 unstructured genes whose protein structures are not available. The functional annotation of up-regulated and down-regulated genes is shown in the Figure $7 \mathbf{a}$ and Figure $\mathbf{7 b}$ respectively. The details of their cellular component functional annotations are shown in Table 3.

Table 1: Confusion matrix or error matrix

\begin{tabular}{lll}
\multirow{2}{*}{ Predicted class } & Actual class & \\
\cline { 2 - 3 } DE & DE & EE \\
EE & True Positive (TP) & False Positive (FP) type I error \\
\hline
\end{tabular}

DE: Differentially Expressed; EE: Equally Expressed; Area under the receiving operating characteristics $(\mathrm{ROC}) \mathrm{curve}, \mathrm{AUC}=$ $(\mathrm{nTPR}+\mathrm{nTNR}) / 2$, Misclassification error rate $(\mathrm{MER})=(\mathrm{nFP}+\mathrm{nFN}) /(\mathrm{nTP}+\mathrm{nTN}+\mathrm{nFP}+\mathrm{nFN})$. 


\section{BIOINFORMATION}

Discovery at the interface of physical and biological sciences

Table 2: Performance Investigation for synthetic dataset

\begin{tabular}{|c|c|c|c|c|c|}
\hline $\begin{array}{l}\text { Performance } \\
\text { Evaluation }\end{array}$ & edge $R$ & SAMSeq & Voom.limma & t-test & Proposed \\
\hline \multicolumn{6}{|c|}{$5 \%$ outliers } \\
\hline Sensitivity & 0.360 & 0.015 & 0.493 & 0.046 & 0.546 \\
\hline Specificity & 0.761 & 0.984 & 0.325 & 0.046 & 0.314 \\
\hline MER & 0.774 & 0.890 & 0.691 & 0.867 & 0.537 \\
\hline FDR & 0.269 & 0.821 & 0.256 & 0.216 & 0.085 \\
\hline AUC & 0.664 & 0.515 & 0.694 & 0.320 & 0.744 \\
\hline pAUC & 0.057 & 0.009 & 0.059 & 0.006 & 0.024 \\
\hline ACC & 0.226 & 0.11 & 0.309 & 0.133 & 0.463 \\
\hline PPV & 0.731 & 0.179 & 0.744 & 0.784 & 0.915 \\
\hline NPV & 0.761 & 0.984 & 0.325 & 0.046 & 0.314 \\
\hline \multicolumn{6}{|c|}{$10 \%$ outliers } \\
\hline Sensitivity & 0.372 & 0.019 & 0.391 & 0.046 & 0.476 \\
\hline Specificity & 0.693 & 0.982 & 0.346 & 0.046 & 0.270 \\
\hline MER & 0.812 & 0.884 & 0.796 & 0.859 & 0.685 \\
\hline FDR & 0.280 & 0.314 & 0.276 & 0.176 & 0.066 \\
\hline AUC & 0.581 & 0.515 & 0.570 & 0.342 & 0.710 \\
\hline pAUC & 0.048 & 0.010 & 0.036 & 0.006 & 0.024 \\
\hline ACC & 0.188 & 0.116 & 0.204 & 0.141 & 0.315 \\
\hline PPV & 0.72 & 0.686 & 0.724 & 0.824 & 0.934 \\
\hline NPV & 0.693 & 0.982 & 0.346 & 0.046 & 0.27 \\
\hline \multicolumn{6}{|c|}{$15 \%$ outliers } \\
\hline Sensitivity & 0.364 & 0.018 & 0.421 & 0.048 & 0.640 \\
\hline Specificity & 0.764 & 0.985 & 0.291 & 0.048 & 0.342 \\
\hline MER & 0.445 & 0.889 & 0.470 & 0.869 & 0.227 \\
\hline FDR & 0.014 & 0.732 & 0.0323 & 0.213 & 0.075 \\
\hline AUC & 0.634 & 0.516 & 0.642 & 0.337 & 0.748 \\
\hline pAUC & 0.055 & 0.010 & 0.047 & 0.007 & 0.029 \\
\hline ACC & 0.555 & 0.111 & 0.53 & 0.131 & 0.773 \\
\hline PPV & 0.986 & 0.268 & 0.9677 & 0.787 & 0.925 \\
\hline NPV & 0.764 & 0.985 & 0.291 & 0.048 & 0.342 \\
\hline \multicolumn{6}{|c|}{$20 \%$ outliers } \\
\hline Sensitivity & 0.405 & 0.017 & 0.439 & 0.046 & 0.612 \\
\hline Specificity & 0.812 & 0.979 & 0.247 & 0.046 & 0.352 \\
\hline MER & 0.702 & 0.885 & 0.652 & 0.878 & 0.216 \\
\hline FDR & 0.058 & 0.217 & 0.055 & 0.366 & 0.069 \\
\hline AUC & 0.742 & 0.510 & 0.724 & 0.333 & 0.745 \\
\hline pAUC & 0.064 & 0.010 & 0.053 & 0.006 & 0.022 \\
\hline $\mathrm{ACC}$ & 0.298 & 0.115 & 0.348 & 0.122 & 0.784 \\
\hline PPV & 0.942 & 0.783 & 0.945 & 0.634 & 0.931 \\
\hline NPV & 0.812 & 0.979 & 0.247 & 0.046 & 0.352 \\
\hline
\end{tabular}

Table 3: Functional annotation of down-regulated genes

\begin{tabular}{llcc}
\hline & Cellular Component (GO) & \\
\hline Pathway ID & Pathway Description & Count in gene set & False discovery rate (FDR) \\
GO:0044424 & Intracellular part & 135 & $8.95 \mathrm{e}-05$ \\
GO:0005622 & Intracellular & 135 & 0.000353 \\
GO:0043226 & Organelle & 128 & 0.000572 \\
GO:0043227 & Membrane-bounded organelle & 122 & 0.00147 \\
GO:0043229 & Intracellular organelle & 119 & 0.0018 \\
GO:0043231 & Intracellular membrane-bounded organelle & 112 & 0.00282 \\
GO:0005737 & Cytoplasm & 107 & 0.00832 \\
GO:0014731 & spectrin-associated cytoskeleton & 3 & 0.00832 \\
GO:0008091 & spectrin & 3 & 0.00984 \\
GO:0043005 & Neuron projection & 19 & 0.0146 \\
GO:0005623 & Cell & 136 & 0.023 \\
GO:0043233 & Organelle lumen & 54 & 0.023 \\
GO:0044464 & Cell part & 135 & 0.0298 \\
GO:0097458 & Neuron part & 21 & 0.0307 \\
GO:0000974 & Prp19 complex & 2 & 0.0439 \\
GO:0032437 & cuticular plate & 2 & 0.0439 \\
\hline
\end{tabular}

ISSN 0973-2063 (online) 0973-8894 (print) 


\section{BIOINFORMATION \\ Discovery at the interface of physical and biological sciences}

(a)

Boxplot for $\mathbf{5 \%}$ Outliers

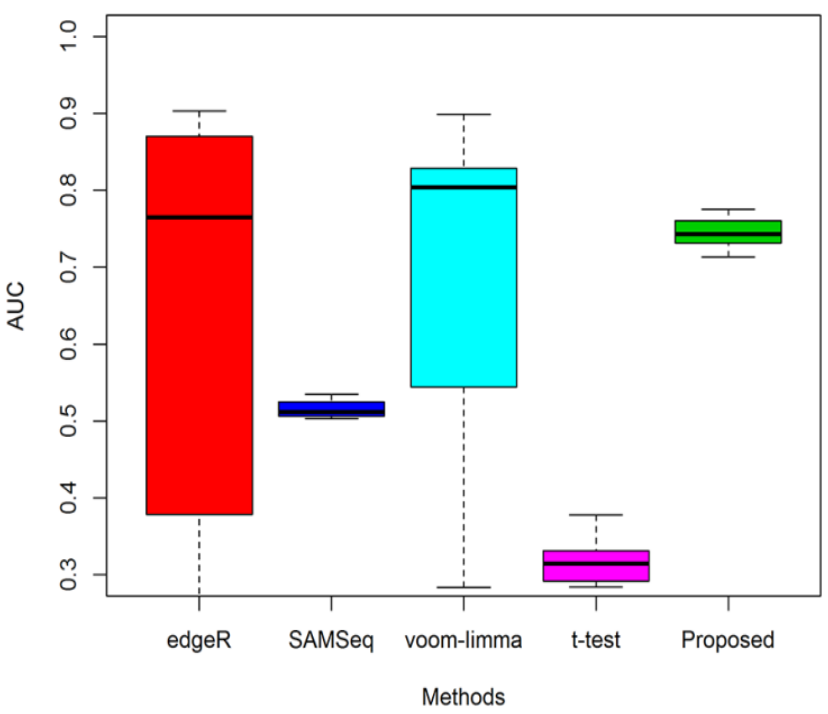

(c)

Boxplot for $15 \%$ Outliers

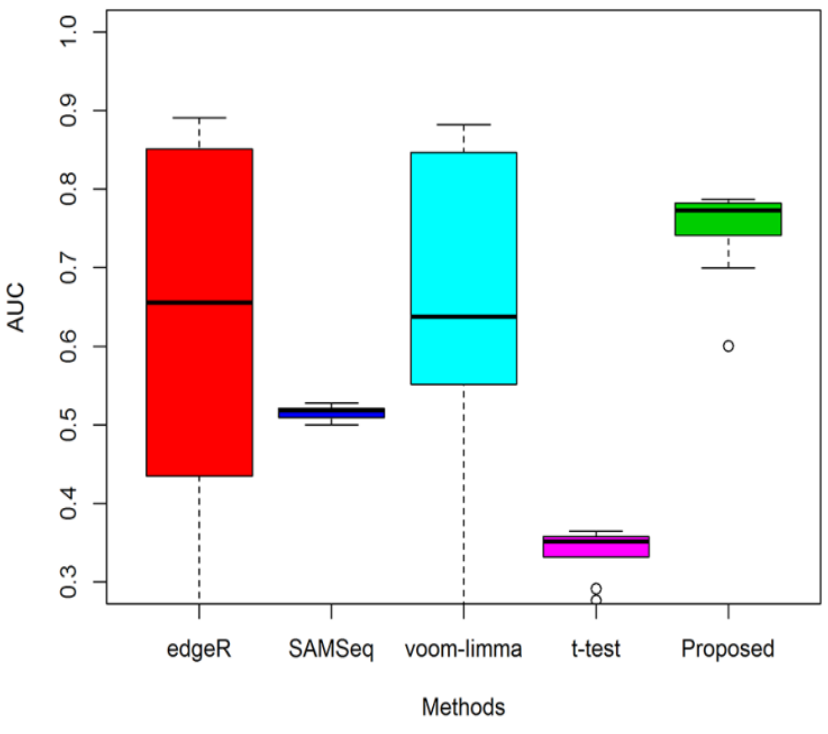

(b)

Boxplot for $10 \%$ Outliers

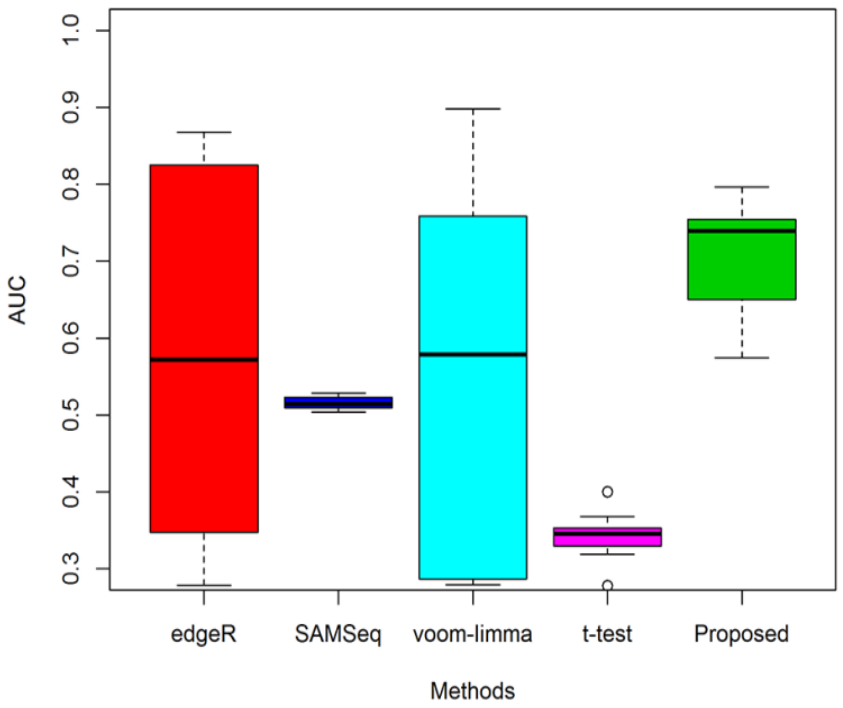

(d)

Boxplot for $\mathbf{2 0} \%$ Outliers

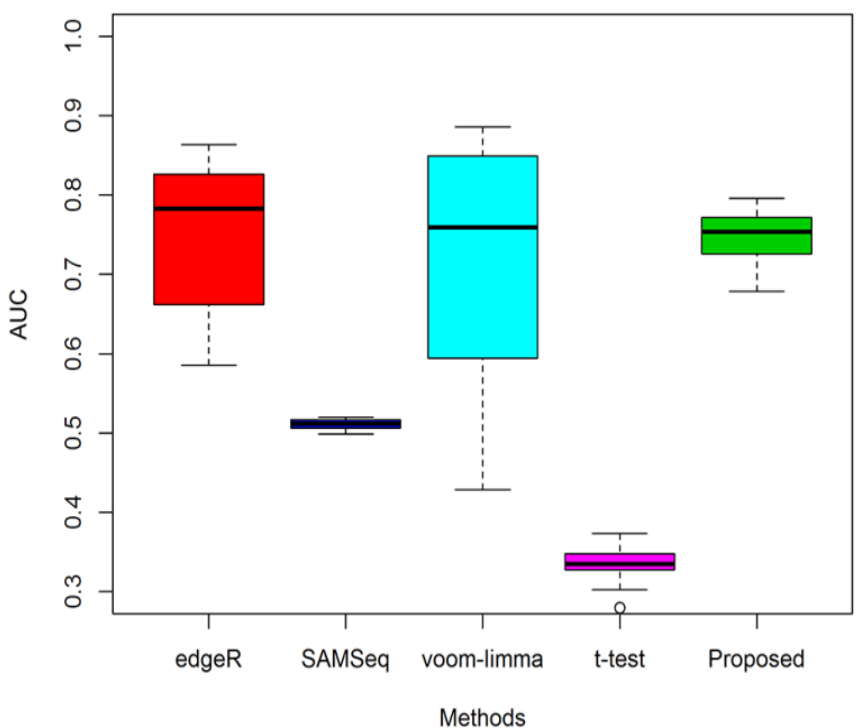

Figure 2: Performance investigation using the boxplot of AUC values of the four methods (edgeR, SAMSeq, voom-limma and proposed) for sample sizes (n1=n2=3). (a) In presence of $5 \%$ outliers (b) In presence of $10 \%$ outliers (c) In presence of $15 \%$ outliers (d) In presence of $20 \%$ outliers.

ISSN 0973-2063 (online) 0973-8894 (print)

Bioinformation 14(4): 153-163 (2018)
BIOMEDICAL

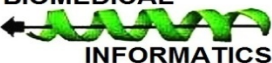




\section{BIOINFORMATION}

Discovery at the interf face of physical and biological sciences (a)

FDR for $5 \%$ Outliers

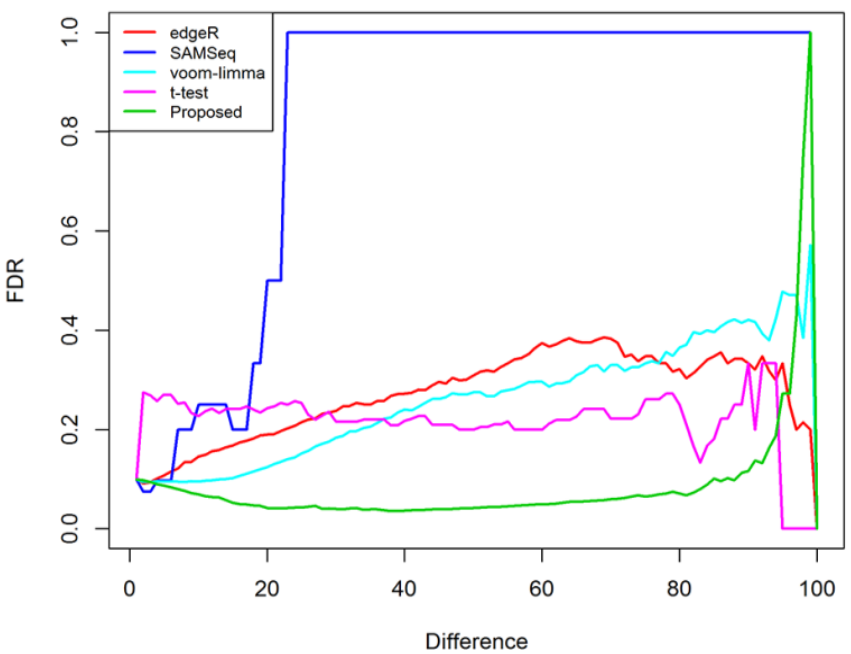

(c)

FDR for $15 \%$ Outliers

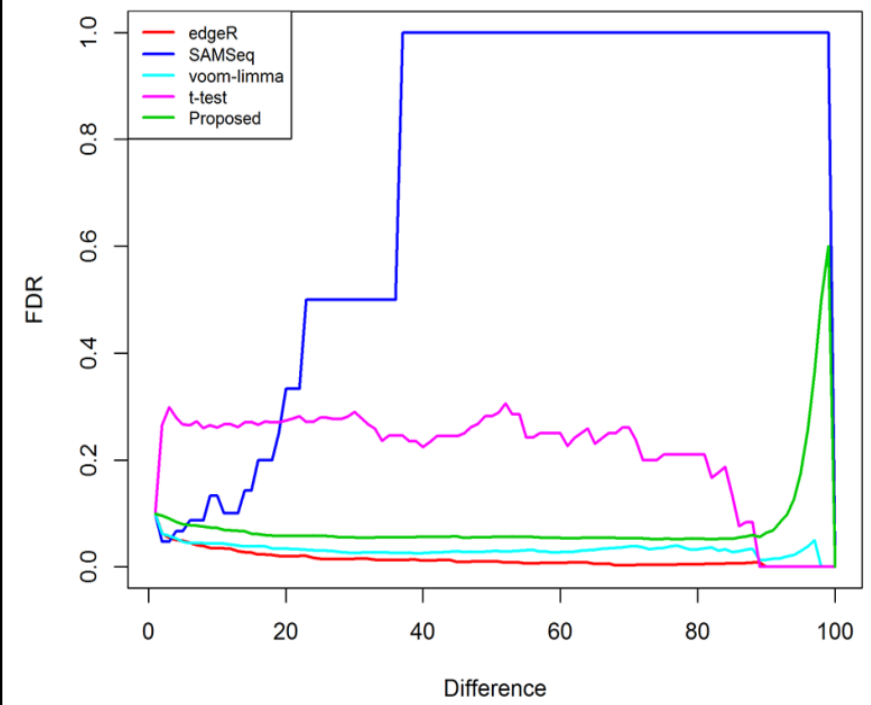

(b)

FDR for $10 \%$ Outliers

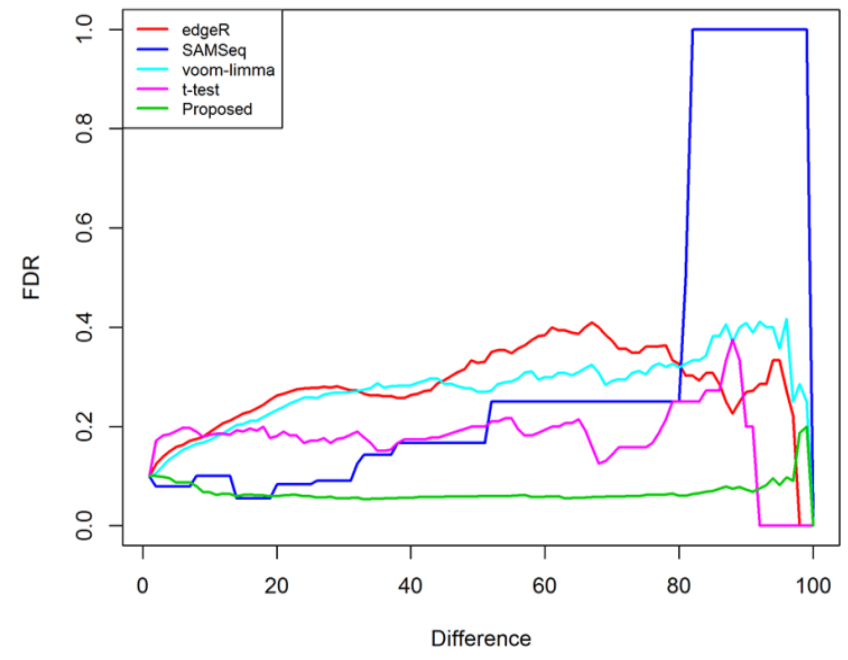

(d)

FDR for $20 \%$ Outliers

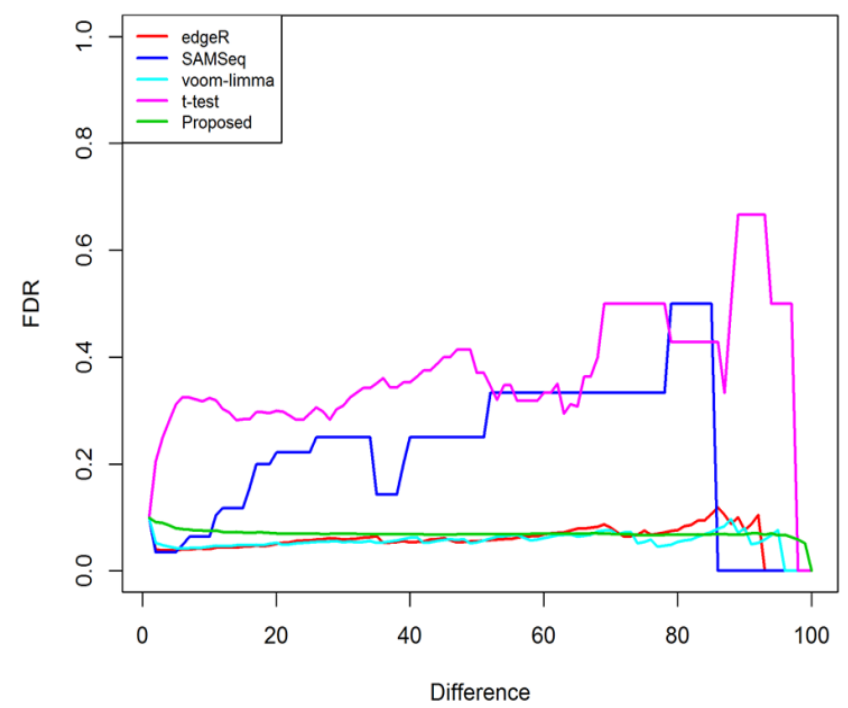

Figure 3: Performance test using FDR plot of the four methods (edgeR, SAMSeq, voom-limma and proposed) for sample sizes $(n 1=n 2=3)$. (a) In presence of $5 \%$ outliers (b) In presence of $10 \%$ outliers (c) In presence of $15 \%$ outliers (d) In presence of $20 \%$ outliers

ISSN 0973-2063 (online) 0973-8894 (print)

Bioinformation 14(4): 153-163 (2018)
BIOMEDICAL

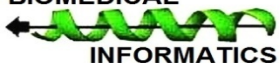

두일 


\section{BIOINFORMATION}

Discovery at the interface of physical and biological sciences

(a)

ROC for $5 \%$ Outliers

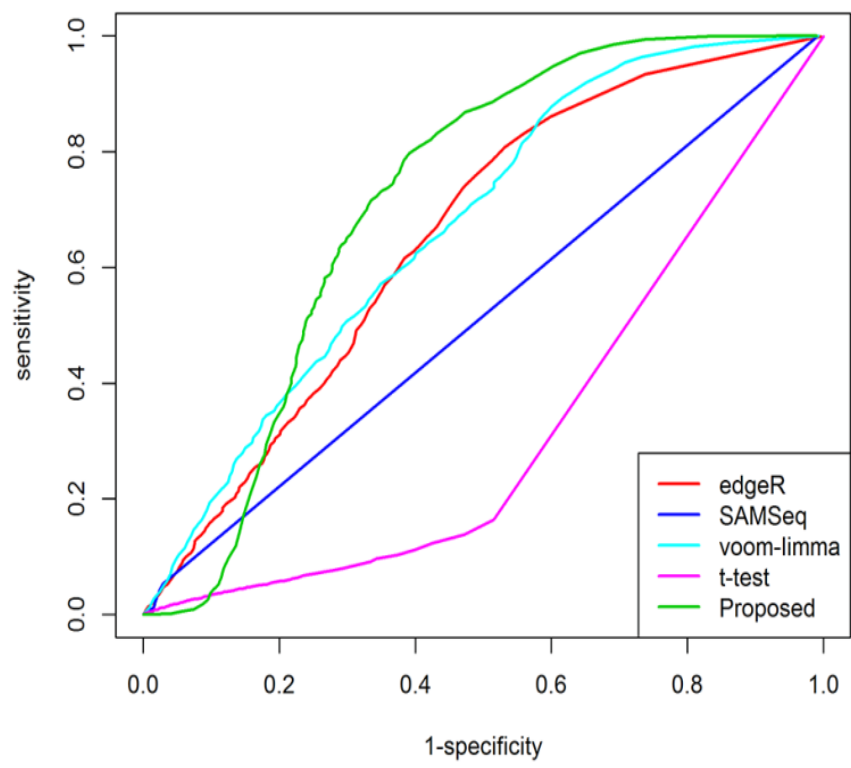

(c)

ROC for $15 \%$ Outliers

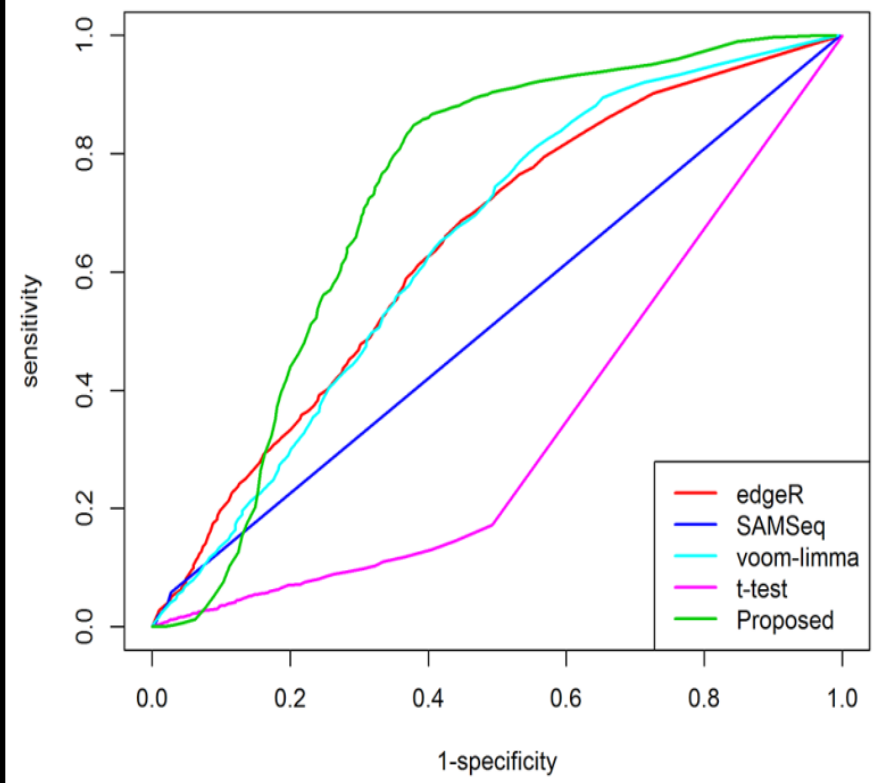

(b)

ROC for $10 \%$ Outliers

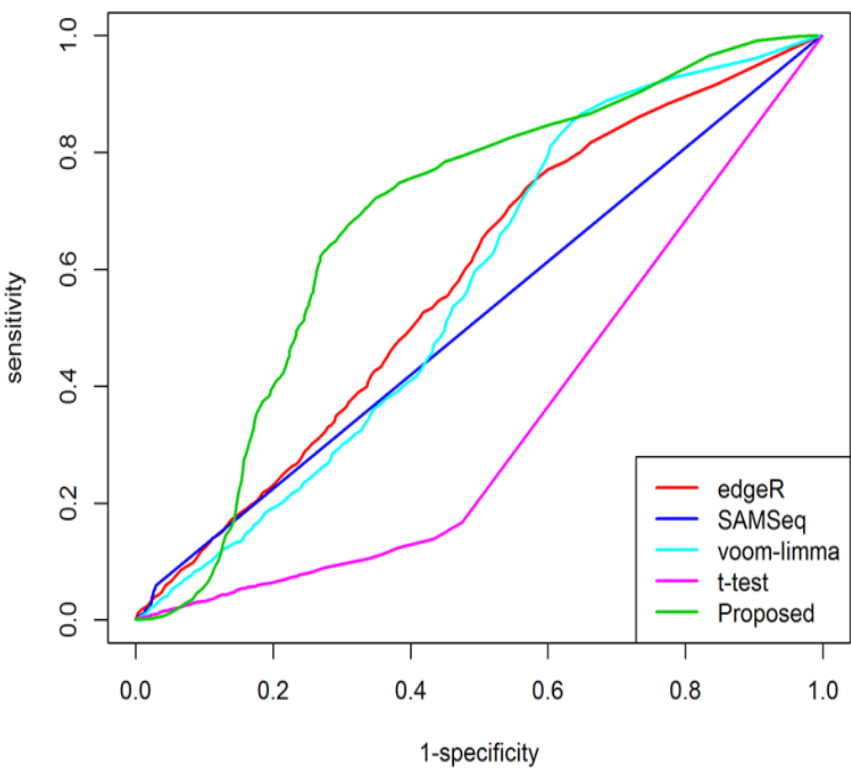

(d)

ROC for $\mathbf{2 0} \%$ Outliers

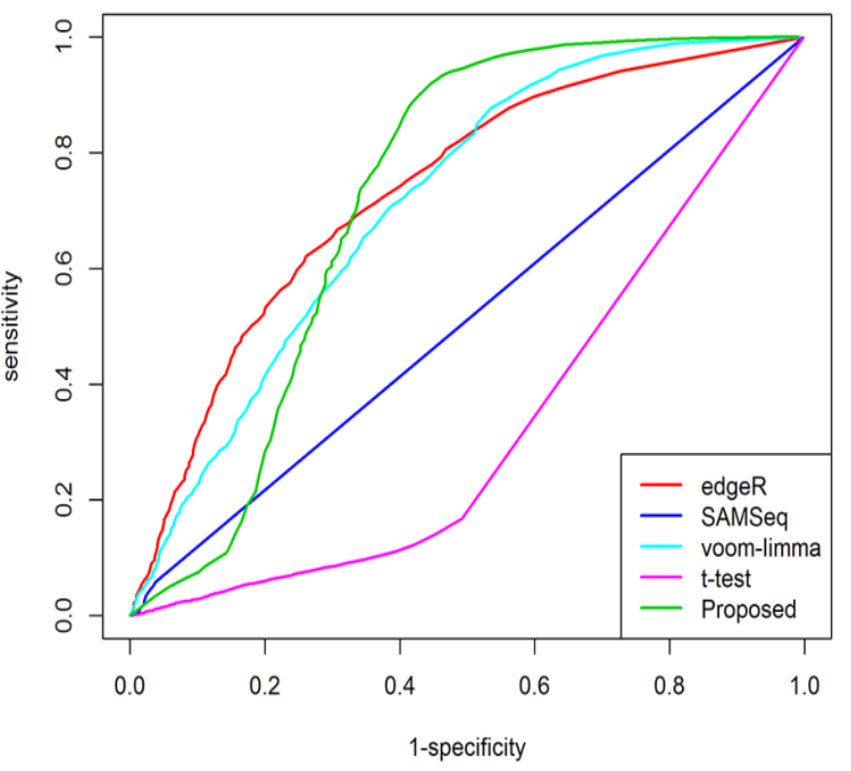

Figure 4: Performance analysis using the ROC of the four methods (edgeR, SAMSeq, voom-limma and proposed). (a) In presence of $5 \%$ outliers (b) In presence of $10 \%$ outliers (c) In presence of $15 \%$ outliers (d) In presence of $20 \%$ outliers.

ISSN 0973-2063 (online) 0973-8894 (print)

Bioinformation 14(4): 153-163 (2018)
BIOMEDICAL

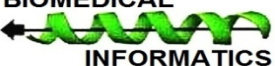




\section{BIOINFORMATION \\ Discovery at the interface of physical and biological sciences}

Open access
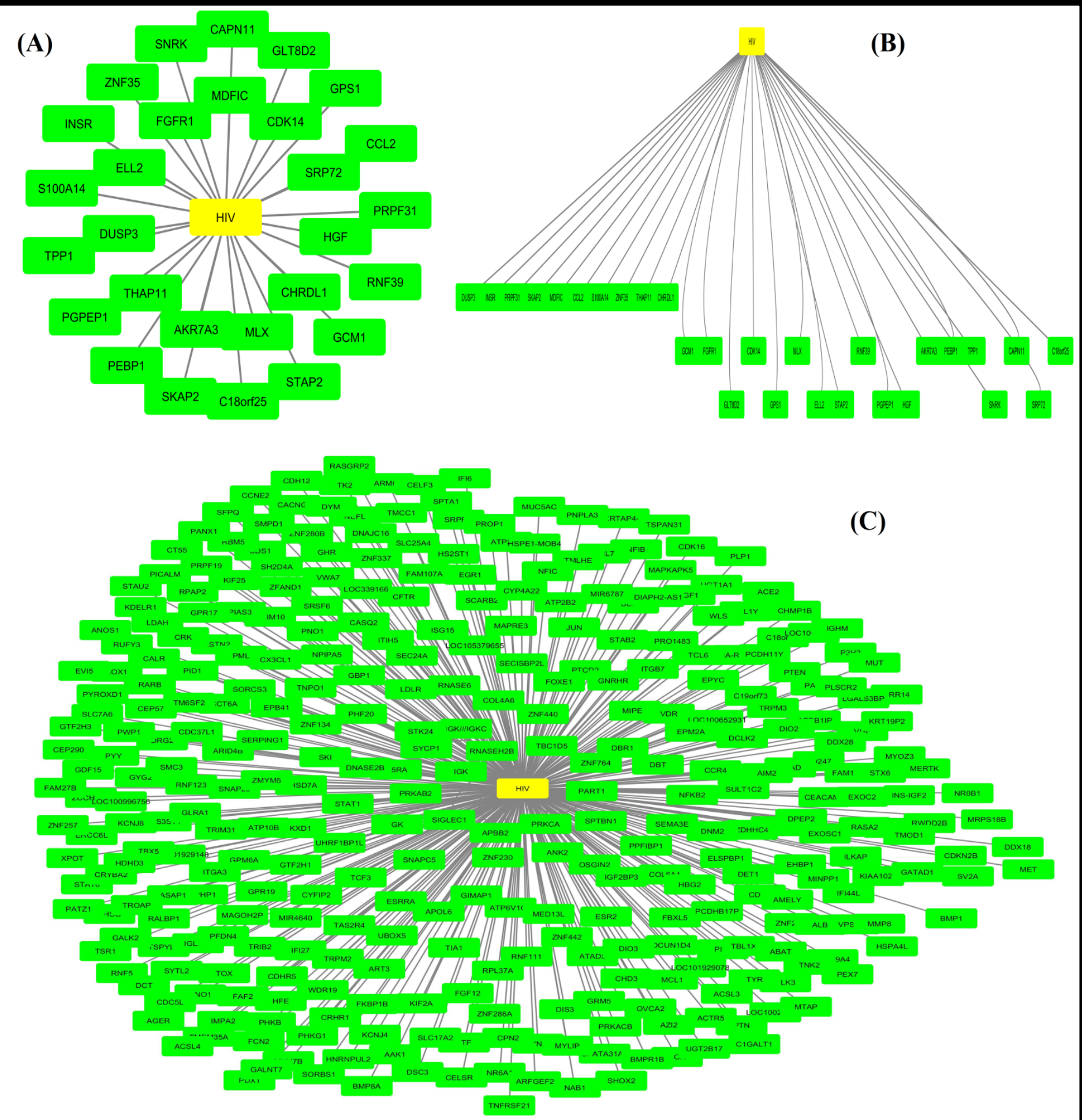

Figure 5: (A) Up-regulated gene-disease network (B) Hierarchical clustering of up-regulated genes (C) Gene-disease network of downregulated genes.

ISSN 0973-2063 (online) 0973-8894 (print)

Bioinformation 14(4): 153-163 (2018)
BIOMEDICAL

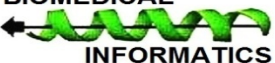




\section{BIOINFORMATION}

Discovery at the interface of physical and biological sciences

(A)

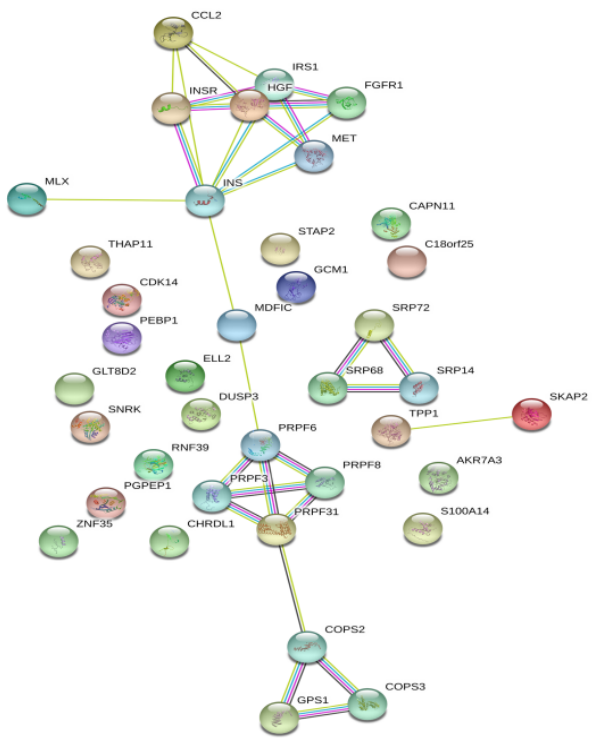

(C)

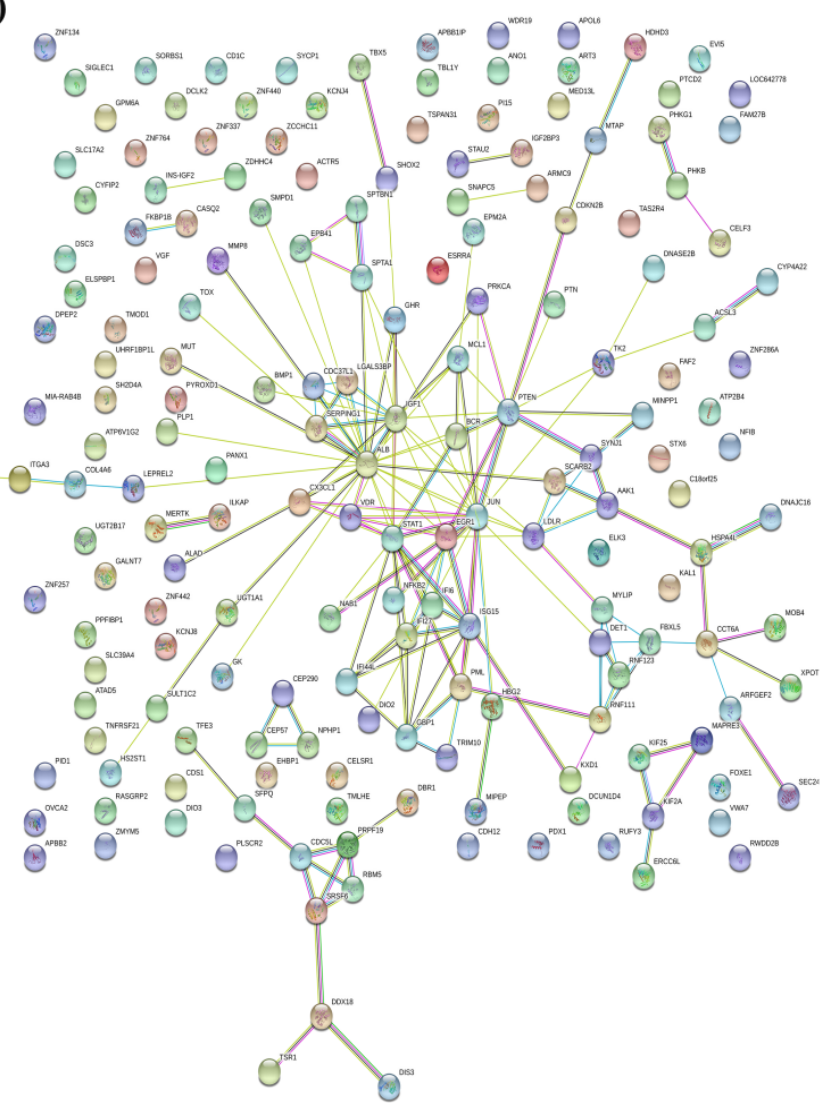

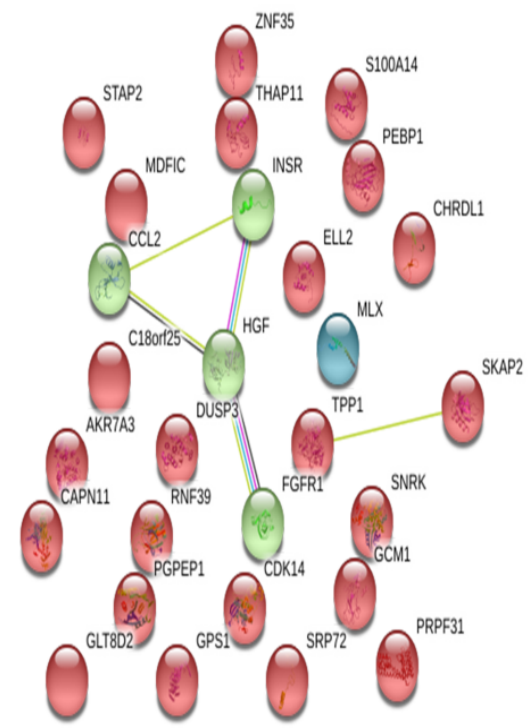

(B)
(D)

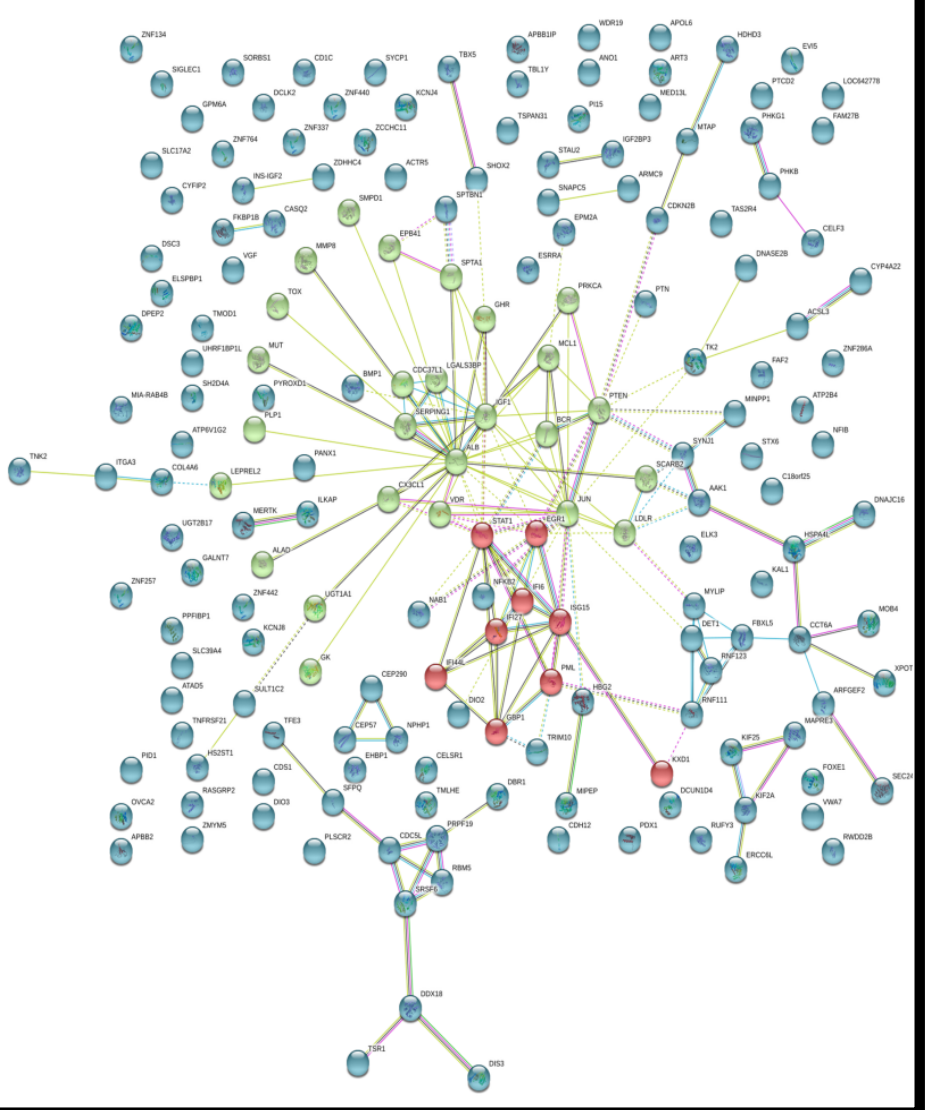

Figure 6: (A) Up-regulated gene protein-protein interaction (PPI) network (B) K-mean clustering of up-regulated genes (C) Downregulated gene PPI networks and (D) K-means clustering of down-regulated genes of HIV disease.

ISSN 0973-2063 (online) 0973-8894 (print)

Bioinformation 14(4): 153-163 (2018)
BIOMEDICAL

- 


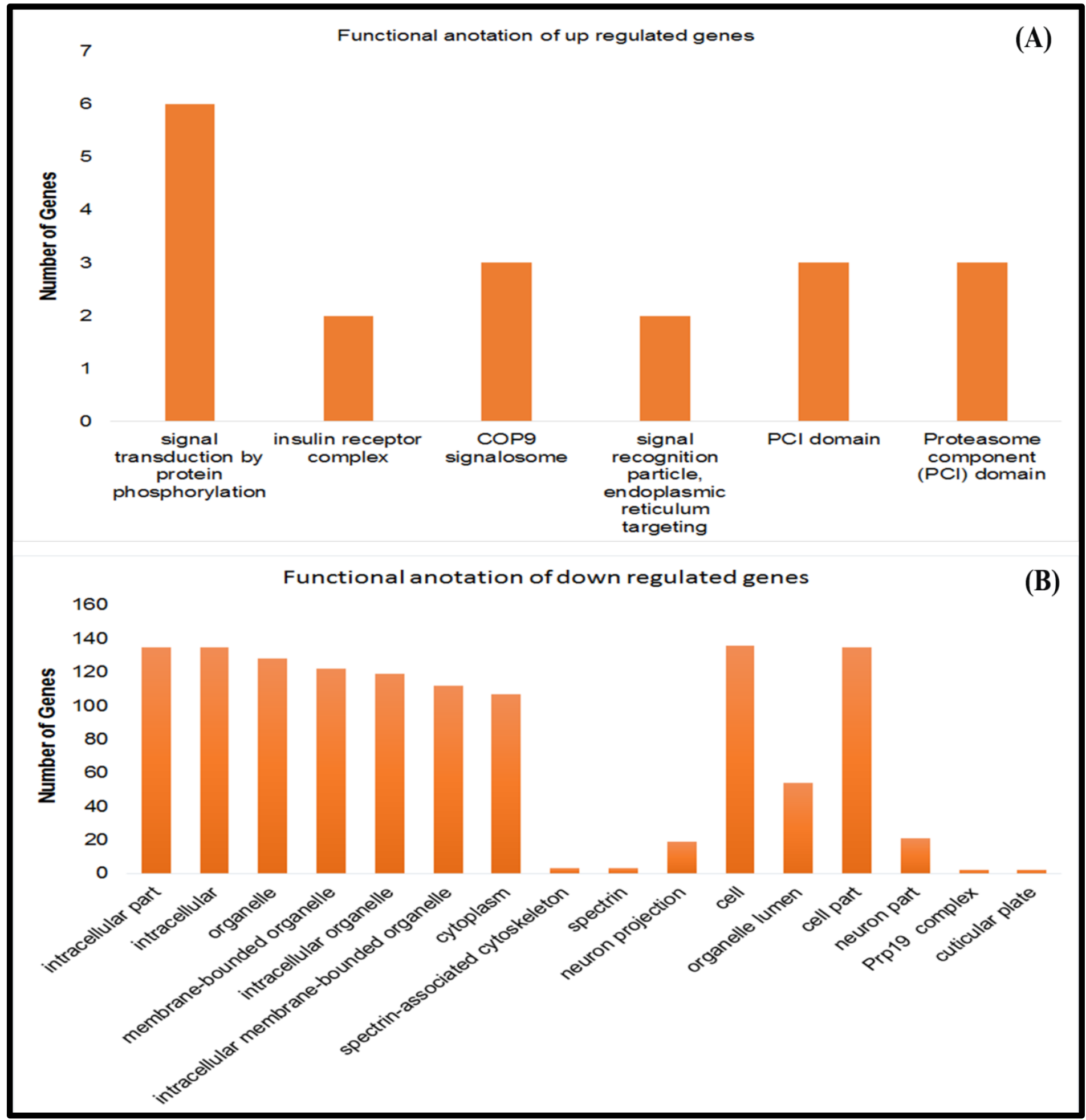

Figure 7: Functional annotation of (A) Up-regulated DE genes (B) Down-regulated genes of real RNA-Seq HIV disease dataset.

ISSN 0973-2063 (online) 0973-8894 (print)

Bioinformation 14(4): 153-163 (2018)
BIOMEDICAL

inFormATICS 


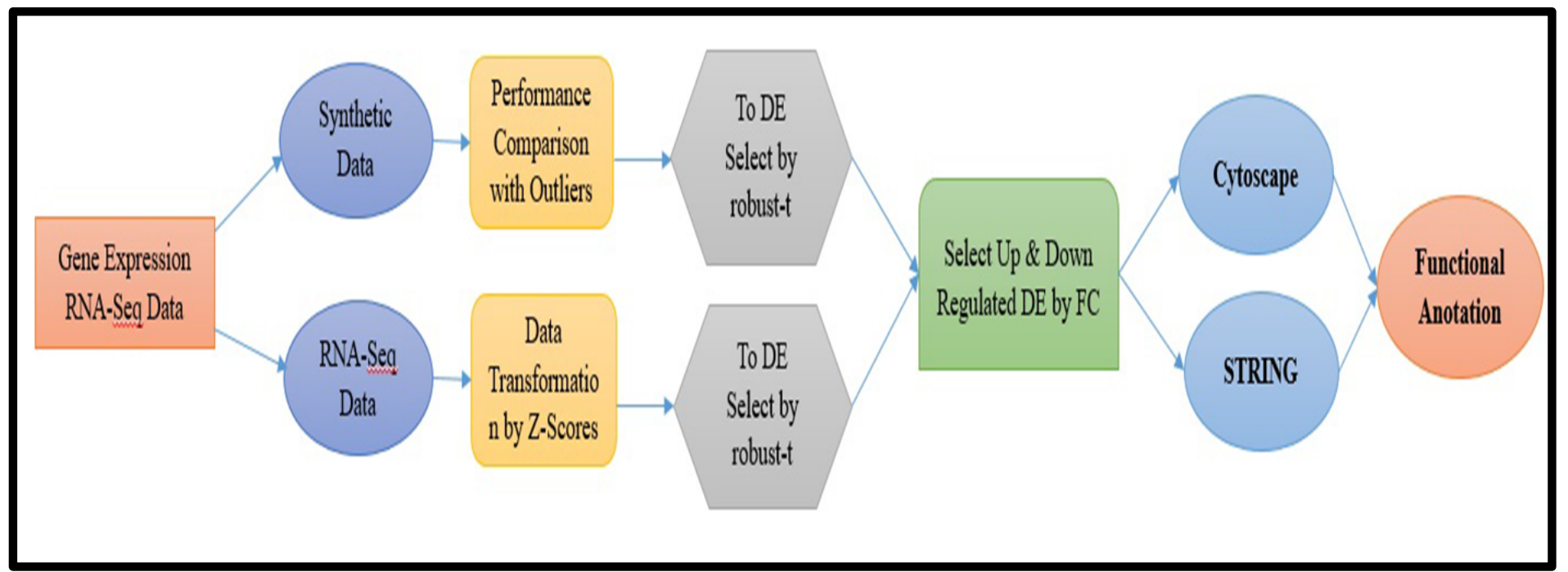

Figure 8: Full work flow of the study paper

\section{Conclusions:}

RNA-seq data analysis helps to identify DEGs to solve important biological problems. Statistical approaches such as edgeR, SAMSeq and voom-limma are widely used in DEGs identification. However, these methods are sensitive to outliers. Hence, we report a robust $t$-statistic using minimum $\beta$-divergence method to overcome this issue. Analysis of synthetic data showed that the robust $t$-statistic method produced better performance with high AUC, low MER and FDR. The real RNAseq count data analysis detected 409 DEG using this method. There are 28 up-regulated genes and 381 down-regulated genes estimated by $\log 2$ fold change (FC) approach at threshold value 1.5. Thus, the identification of potential biomarkers from RNAseq dataset using a robust $\mathrm{t}$-statistical model is demonstrated.

\section{References:}

[1] David S et al. Biomarkers in Toxicology.

[2] Dillies MA et al. Briefings in Bioinformatics. 2012, 14:671. [PMID: 22988256].

[3] Wang et al. Nature. 2008, 456:470. [PMID: 18978772].

[4] Pan Q et al. Nature Genetics. 2008, 40:1413. [PMID: 18978789].

[5] Landau et al. PLoS ONE. 2013, 8:e81415.
[6] Soneson C \& Delorenzi MA. BMC Bioinformatics.2013, 14:91.

[7] Tang et al. BMC Bioinformatics. 2015, 16:361.

[8] Oshlack A et al. Genome Biology. 2010. 11:220.

[9] Agarwal et al. BMC Genomics. 2010. 11:383 [PMID: 2056764]

[10] Bradford JR et al. BMC Genomics. 2010, 11:282.

[11] Robinson MD et al. Bioinformatics. 2010, 18:1509 [PMCID: PMC2796818]

[12] Li J and Tibshirani R. Statistical Methods in Medical Research. 2013. [PMID: 22127579]

[13] Ander S and Huber W. Genome Biology. 2010, 11:94 [PMCID: PMC3218662]

[14] Smyth GK. Statistical Applications in Genetics and Molecular Biology. 2004, 3. [PMID: 16646809]

[15] Mollah et al. Neural Networks. 2010, 23:226.

[16] Dembélé D and Kastner P. BMC Bioinformatics. 2014, 15:14 [PMID: 24423217].

[17] Tilton JC et al. Journal of Virology. 2006, 80:11486 [PMID: 17005663].

[18] Shannon P et al. Genome Research. 2003, 13:2498 [PMCID: PMC403769]

[19] Szklarczyk D et al. Nucleic Acids Research. 2017, 45:D362 [PMID: 27924014]

Edited by $P$ Kangueane

Citation: Akond et al. Bioinformation 14(4): 153-163 (2018) License statement: This is an Open Access article which permits unrestricted use, distribution, and reproduction in any medium, provided the original work is properly credited. This is distributed under the terms of the Creative Commons Attribution License

\section{ISSN 0973-2063 (online) 0973-8894 (print)}

Bioinformation 14(4): 153-163 (2018) 